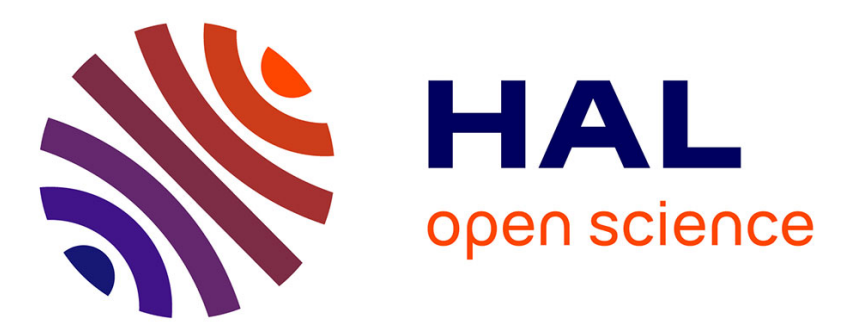

\title{
Control of particle morphology in the spray drying of colloidal suspensions
}

E. Lintingre, F. Lequeux, Laurence Talini, N. Tsapis

\section{To cite this version:}

E. Lintingre, F. Lequeux, Laurence Talini, N. Tsapis. Control of particle morphology in the spray drying of colloidal suspensions. Soft Matter, 2016, 12 (36), pp.7435-7444 10.1039/C6SM01314G . hal-01368558

\section{HAL Id: hal-01368558 \\ https://hal.sorbonne-universite.fr/hal-01368558}

Submitted on 19 Sep 2016

HAL is a multi-disciplinary open access archive for the deposit and dissemination of scientific research documents, whether they are published or not. The documents may come from teaching and research institutions in France or abroad, or from public or private research centers.
L'archive ouverte pluridisciplinaire HAL, est destinée au dépôt et à la diffusion de documents scientifiques de niveau recherche, publiés ou non, émanant des établissements d'enseignement et de recherche français ou étrangers, des laboratoires publics ou privés. 


\title{
Control of particle morphology in the spray drying of colloidal suspensions
}

\author{
E. Lintingre, ${ }^{a, b}$ F. Lequeux, ${ }^{a, b}$ L. Talini ${ }^{a, b}+$ and N. Tsapis ${ }^{c}$
}

Powders of nanoparticles are volatile, i.e. easily disperse into air, which makes their handling difficult. Granulation of nanoparticle powders provides a solution to that issue, and it is generally performed by spray drying the nanoparticles that have been suspended in a liquid. Spray drying a colloidal suspension consists in atomising the suspension into droplets by a fast flowing and hot gas. Once the droplets dried, the resulting dry grains/microparticles can be used in a wide range of applications - food, pharmaceutics, fillers, ceramics, etc... . It is well known that the grains resulting from spray-drying may be spherical but may also exhibit other diverse morphologies. Although different influencing parameters have been identified, no clear overview can be found in the literature for the driving mechanisms of grain shaping. In the present work, we review the assumptions made in the literature to explain the different morphologies. We analyse the orders of magnitude of the different effects at stake and show that the grain shape does not result from a hydrodynamic instability but is determined by the drying stage. However, we emphasize that neither the drying time nor the associated Péclet number are critical parameters for the determination of shape morphology. In the light of those results, we also review and discuss the single droplet experiments developed to mimic spray drying. Generalising our previous works, we further analyse how the control of morphology can be achieved by tuning the colloidal interactions in the suspension. We detail the model we have developed that relates the colloidal interaction potential to a critical pressure exerted by the solvent as it flows, and we provide a quantitative prediction of the grain shape. Finally, we offer perspectives with regard to spray drying of systems such as molecular solutions, widely performed in e.g. the pharmaceutical industry.

\section{Introduction}

Dry powders of nanoparticles are prone to volatility and consequently difficult to handle. Volatility is a major concern in industry since, in particular, workers may inhale nanoparticles, which potential toxicity remains an open question. ${ }^{1}$ For easier handling and reduction of safety concerns, a frequently adopted solution consists in granulating nanoparticles. Granulation is usually obtained by spray drying a nanoparticle suspension. In a first step, nanoparticles are suspended into a liquid and formulation is generally required to control the $\mathrm{pH}$ or the ionic strength of the suspension and keep it fluid enough. For that purpose, additives such as surfactants may be used. The nanoparticle suspension is further injected into a chamber and maintained under a flow of hot gas. The injection is performed using injectors or nozzles, which break the suspension into small droplets of typical diameter of 100 micrometers further dried in contact with the hot gas flow. At the end of the process, a powder is recovered made of dry solid grains/microparticles large enough to avoid volatility. The chemical nature of the powders thus transformed varies widely since spray drying is commonly used in the food industry, but also for pharmaceutics, detergents, cosmetics or ceramics applications.

The aim of the present review is to provide an understanding of the mechanisms that may impact the shape of the spray dried grains/microparticles; we focus on grains resulting from the drying of colloidal suspensions formed by dispersing the nanoparticles into a liquid. Although, filled homogenous spheres may form during the spray-drying process, many other grain/microparticle morphologies have been reported, such as doughnut-like or deflated balloons ${ }^{2}$ (see fig. 1 for examples). The grain/microparticle morphology is indeed crucial for some applications. For instance it controls the porosity of the final ceramics formed and hence their mechanical properties. ${ }^{3,4}$ For pharmaceutical applications, morphology requirements may differ according to the route of administration of the powder. If the powder is intended to be placed in a capsule for oral administration, one may want to obtain spherical dense microparticles. ${ }^{5}$ If the spray dried powder is intended for inhalation, crumpled paper morphologies or hollow spheres might be convenient for deeper lung deposition. ${ }^{6}$ Optimization of the powder for its final application therefore requires the full determination and control of the relevant parameters that will allow one to finely tune grain/microparticle morphology.

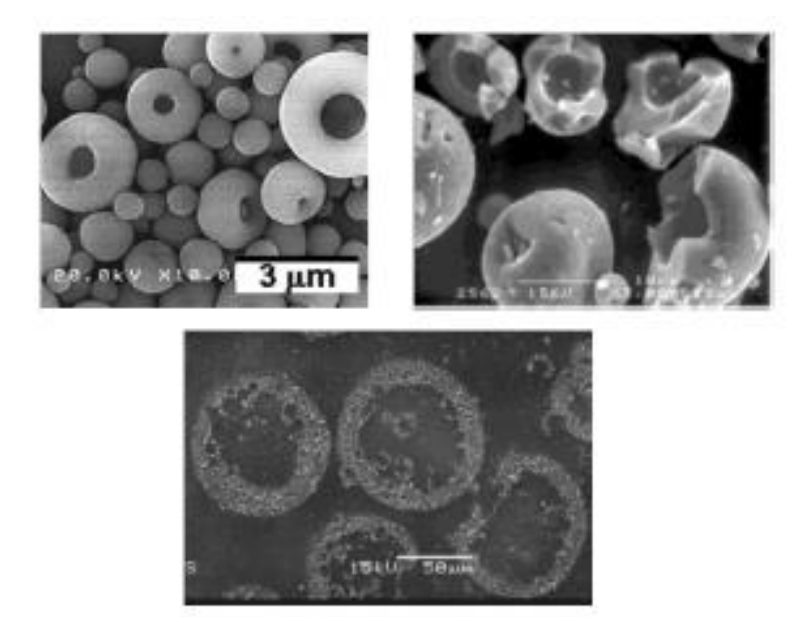

Fig. 1: Examples of grain morphologies resulting from the atomisation of colloidal suspensions (a) doughnut-like from ref ${ }^{7}$ and ( b) and (c) deflated balloons respectively from ref $^{8}$ and ref. $f^{9}$. All figures reprinted with permission.

In practice, a great number of parameters have been tested and found to influence the grain/microparticle morphology. Among them, process parameters, such as gas temperature, 
gas and liquid injection rates and shape of the nozzle have proven relevant. ${ }^{7,10}$ The physico chemical properties of the colloidal suspensions have also been shown to play a major part in the formation of non-spherical grain/microparticle shapes. ${ }^{7,11}$ Despite the importance of spray drying in many fields and the abundant literature on the topic, no clear overview on the role of these parameters is available, in particular because no convincing conclusion is drawn on the physical effects responsible for the different shapes. The problem is actually complex by nature: it combines the hydrodynamics of jets with the drying processes of colloidal suspensions, during which colloidal interactions can play a crucial role.

Our contribution will consist in reviewing the main assumptions made in the literature to explain the morphologies that result from spray drying of colloidal suspensions. On that basis, we analyse the different transfers and flows occurring during spray drying and discuss how they may impact the final grain/microparticle shape. We thus shed new light on the physical effects determining the final grain/microparticle morphology. In the first section, we consider the hypothesis that the morphology is determined by hydrodynamic effects during spray drying. We detail that effect and discuss the relevant dimensionless numbers. Based on this analysis, we show that in most spray dryers, hydrodynamic effects are not at the origin of the non spherical morphologies. Second, the drying process of the suspension droplets has been invoked in order to explain the shape instability. During that process, particles accumulate at the droplet surface, which can lead to the formation of a shell that may further buckle. In the second section, we will show that this description is fully relevant, in contrast to the discussion sometimes found in the literature in terms of Péclet number. In a third section, we will detail how the competition between the colloidal interactions between particles and the Darcy pressure quantitatively accounts for the shape instability of droplets during drying. We will eventually show what practical parameters are relevant and how the morphologies can be fully controlled.

\section{Hydrodynamic effects}

During the spray drying process, a liquid is injected in a gas flow, as schematised in fig. 2. The liquid jet breaks into droplets, and this complex and rich phenomenon has been described in the literature. ${ }^{12,13}$ This is however out of the scope of this review, and we focus on the stage at which the liquid droplets are formed and may later deform. As shown in fig. 2, different time scales must be considered.

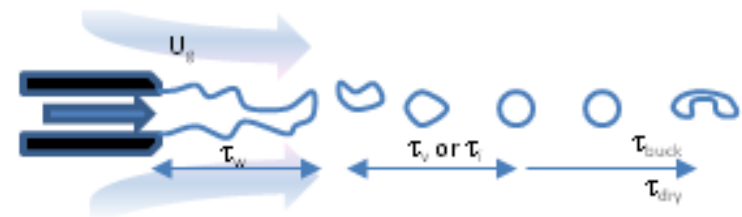

Fig. 2: Schematised atomisation process: the liquid is injected in a gas flow of velocity $\mathrm{U}_{\mathrm{g}}$, with a relative velocity between liquid and gas of initial value $U$. The relative velocity decays to zero during the characteristic time $\tau_{W}$, and the droplet recovers a spherical shape within a time $\tau_{v}$ or $\tau_{i}$ depending on the relative values of viscous and inertial forces. The droplet further dries in a time $\tau_{d r y}$, and may buckle, according to the relative values of $\tau_{d r y}$ and $\tau_{\text {buck }}$.

First, because the relative velocity between the gas and the droplets is not zero, the gas exerts a dynamic pressure on the droplets, which may lead to various shapes and breaking modes of the droplets that have been discussed in the literature. ${ }^{14}$ If the drying time is small compared to the time needed for a deformed shape to relax, then the dried grain will exhibit a non spherical shape. Examples are shown in fig. 3 among which the so-called Pele's tears, i.e. droplets of lava blown during a volcanic eruption that are deformed by the relative air flow, and solidify in a non spherical shape. ${ }^{15}$ The same mechanism has been invoked in the literature as a possible explanation for the non spherical grains obtained from spray-drying processes. ${ }^{7}$ In agreement with recent observations, ${ }^{11}$ we demonstrate in the following that, in usual spray-drying conditions, that mechanism is not responsible for the non spherical shape of the grains. We analyse the different effects at stake, and support our demonstration with quantitative arguments based on new experimental data.

The relevant dimensionless number here is the ratio between the dynamic pressure exerted by the gas, $\rho_{g} U^{2} / 2$, and the Laplace pressure that tends to keep the droplet spherical, $2 \gamma / R$, where $\rho_{g}$ and $\gamma$ are respectively the gas density and the liquid/gas interfacial tension, $R$ the radius of the droplet and $U$ the relative velocity of the droplet with gas. The associated dimensionless number is the Weber number, which writes as: $W e=\rho_{g} U^{2} R / \gamma$. When $W e$ is large compared to unity, the spherical shape becomes unstable.

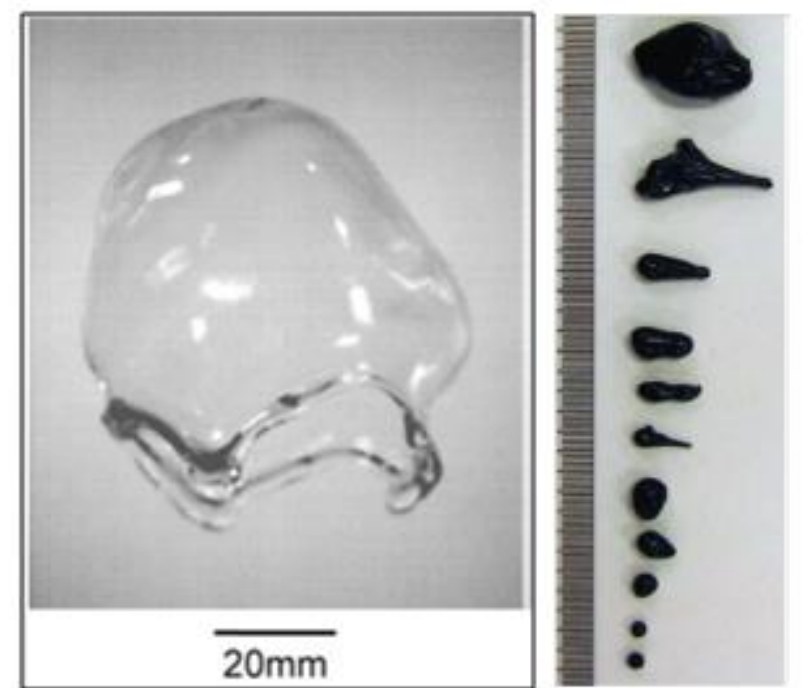

Fig. 3: Examples of drops deformed by a relative gas flow: (a) free-falling water drop in air $^{14}$ and (b) solidified droplets of lava after a volcanic eruption, the largest ones exhibiting shapes of so-called Pele's tears. ${ }^{15}$ Reprinted with permission.

The instability results in fragmentation into smaller droplets. The critical relative velocity corresponding to $W e=1$ writes as: 
$U_{w}=\sqrt{\gamma / \rho_{g} R}$. For a water droplet of $100 \mu \mathrm{m}$ diameter in an air flow, $U_{w}$ is close to $20 \mathrm{~m} . \mathrm{s}^{-1}$. Under that velocity value, the spherical shape of the droplet is stable.

Indeed, in the spray dryer, the relative velocity of the gas at the exit of the nozzle is such that droplets of the required diameter are obtained. Once formed, the droplets further accelerate, and the relative velocity with the gas, and thus the Weber number, decreases with time. In the limit of large Weber numbers, corresponding to the initial times of the droplet motion, the time needed for the decay of the relative velocity can be estimated by writing a balance of the forces per unit of volume exerted on the droplet:

$$
\frac{4}{3} \Delta \rho \pi R^{3} \frac{d U}{d t} \approx \rho_{g} \pi R^{2} \frac{U^{2}}{2}
$$

where $\Delta \rho$ is the density difference between the droplet and the air.

The left-hand side of eq. (1) corresponds to the buoyant mass of the droplet times its acceleration, and the right-hand side to the hydrodynamic force exerted on the droplet. Assuming that $d U / d t \approx U / \tau_{W}$, the resulting estimate for the decay time is $\tau_{W} \sim 8 R \Delta \rho / 3 \rho_{g} U$.

Once that time elapsed, it is expected that the gas flow induces no further shape instability- provided the gas turbulence does not result in a new relative velocity. That last hypothesis is sound since the atomization process is not modified by turbulent velocity fluctuations in the liquid nor in the gas. ${ }^{13}$ An estimate for the maximum value of $\tau_{W}$ is obtained for a velocity equal to the Weber velocity $U_{w}$, yielding $\tau_{W} \sim 10 \mathrm{~ms}$ for a water droplet in air. Therefore, the forces created by the gas flow heterogeneity cannot sustain for a time longer than $10 \mathrm{~ms}$ on the droplet. After that time, the droplet rapidly recovers its spherical shape. The time needed for that process is given by the balance of the capillary forces and either the viscous or inertial forces inside the droplet. The corresponding relaxation times are $\tau_{v}=\mu R / \gamma$ where $\mu$ is the viscosity of the liquid in the droplet and $\tau_{i}=\left(\rho R^{3} / \gamma\right)^{1 / 2}$ where $\rho$ is the liquid density

For a water droplet of diameter $100 \mu \mathrm{m}, \tau_{v}$ is smaller than $1 \mu \mathrm{s}$ and $\tau_{i}$ is smaller than $100 \mu \mathrm{s}$. Obviously, $\tau_{v}$ increases with viscosity, and can become larger than $\tau_{i}$, but, even for a viscosity four orders of magnitude larger than the one of water, the relaxation time remains smaller than $10 \mathrm{~ms}$. Larger values are expected for droplets of suspensions that are more viscous or that exhibit a yield stress larger than about $100 \mathrm{~Pa}$. However, the atomization of such suspensions is prohibited since the injection stage requires liquids of low viscosity. Therefore, in usual spray-drying conditions, the droplet deformation induced by gas pressure gradient only lasts for times smaller than $10 \mathrm{~ms}$. That estimate is in good agreement with experimental values reported in the literature, showing that $W e=1$ is reached within a few milliseconds. ${ }^{12}$ We show in the following section that the drying process is much slower than the formation of spherical droplets.

\section{The drying stage}

Once a droplet is formed in the spray dryer, the solvent - in general water -evaporates in the hot gas flow. The limiting step for drying is the heat and solvent exchange between the droplets and the surrounding gas. Evaporation induces a temperature decrease at the surface of the droplet, which is balanced by the heat flux from the hot gas. Assuming the temperature difference $\Delta T$ between the solvent boiling temperature and the gas is confined within a layer of thickness $\delta$, the balance writes as:

$$
\Delta H \rho_{\text {liq }} \frac{\partial\left(\frac{4 \pi}{3} R^{3}\right)}{\partial t}=\lambda_{\text {gas }} \frac{4 \pi R^{2} \Delta T}{\delta}
$$

where $\Delta H$ is the vaporisation latent heat of the solvent, $\rho_{\text {liq }}$ its density, $\lambda_{\text {gas }}$ the thermal conductivity of the gas, $R$ the droplet radius. The thickness $\delta$ depends on the relative velocity of the droplet with the gas, but is expected to remain smaller than the droplet radius. The characteristic time for the drying of the droplet is simply given by:

$$
\tau_{d r y_{f} f l u x} \cong \frac{\Delta H \rho_{\text {liq }}}{\lambda_{\text {gas }} \Delta T} R \delta
$$

The other mechanism that is likely to limit the drying is the diffusion of solvent molecules in air. In that case, the drying time writes as:

$$
\tau_{d r y_{\_} \text {diff }} \cong \frac{\rho_{\text {liq }}}{D_{\text {gas }} c_{\text {sat }}} R \delta
$$

Where $D_{\text {gas }}$ and $c_{\text {sat }}$ are respectively the diffusion coefficient and saturation concentration of solvent in gas. As the droplet contains colloidal particles, the saturation concentration of the solvent is expected to depend on their osmotic pressure. However, that effect is negligible in usual conditions. ${ }^{\ddagger}$

Numerically, the times given by eq. (3) and eq. (4) are of the same order of magnitude with, for water, respectively $\tau_{d r y} / R \delta \cong 6.10^{8} \mathrm{~s} . \mathrm{m}^{-2}$, with $\Delta T=100 \mathrm{~K}$, and $\tau_{d r y} / R \delta \cong$ $10^{9} \mathrm{s.}^{-2}$ in the diffusion limited drying. Those times are of the order of a few seconds for $100 \mu \mathrm{m}$ droplets.

As a consequence, the drying time is at least one order of magnitude greater than the time needed to form spherical droplets, definitely confirming the non spherical shape of the grains does not result from hydrodynamic effects. The grain morphology therefore results from phenomena at stake in the drying stage of the droplets of colloidal suspensions. Several model experiments have been developed in order to reproduce drying conditions in atomizers and study that stage more easily; we briefly review the different principles of those set-ups in the following section.

\section{Single droplet experiments to mimic spray drying}

To better understand the origin of non spherical grains/nanoparticles, several experimental set-ups have been proposed to study the drying of single droplets in conditions close to those found in a spray-dryer. One can observe a droplet drying at the tip of a glass fiber. ${ }^{16}$ The mass of the 
droplet can be monitored during the drying process by connecting the fibre to a mass balance, while the morphology can be observed by a camera. Of course, the contact with the fibre induces heat transfer in addition to the one due to a hot gas flow. Alternatively, droplets can be deposited on a hydrophobic flat surface and submitted to hot gas flow. ${ }^{17}$ Very small droplets can be observed (down to $150 \mu \mathrm{m}$ in diameter) and high throughput systems have been developed to dry several droplets simultaneously. One should however note that the presence of the hydrophobic surface influences both the air temperature and the hot gas flow pattern in the vicinity of the droplet, thereby reducing the drying rate.

Different levitation techniques have also been proposed to study the drying process. The first one is acoustic levitation where a sound wave is applied on a droplet from $100 \mu \mathrm{m}$ to $2 \mathrm{~cm}$ diameter in a closed chamber while heating is achieved through laser beams. ${ }^{18-20}$ This set-up has the limitation of inducing droplet shape distortion due to air drag or acoustic forces. ${ }^{21}$ In addition, the acoustic field may modify the heat and mass transfer as compared with hot gas convection. ${ }^{19}$ This system is therefore far from realistically mimicking the spray drying conditions. The second levitation method is aerodynamic levitation where the droplet is submitted to a vertical gas jet to counterbalance gravity. ${ }^{21}$ Stability of the droplet is a concern with this set-up as the gas flow should be adapted to the change in droplet mass as it evaporates. In addition, as the droplet solidifies and potentially deviates from sphericity the stability in the gas stream may be difficult to maintain.

A simple method to observe a single droplet drying has been recently suggested, which is based on the Leidenfrost effect: ${ }^{23}$ a liquid droplet deposited on a sufficiently hot surface floats on a thin layer of its own vapour. Provided the droplet radius is smaller than the capillary length, surface tension dominates over gravity and one obtains spherical droplets with evaporation occurring on the whole surface and not by exchange with the vapour layer. ${ }^{24}$ For small Leidenfrost droplets, the evaporation process therefore reproduces the main features of the spray drying process. In particular, the same morphologies as the ones obtained in atomization are observed (fig. 4). This set-up was applied to study the drying mechanism of colloidal suspensions with an excellent correlation between Leidenfrost and spray drying results. ${ }^{25-27}$
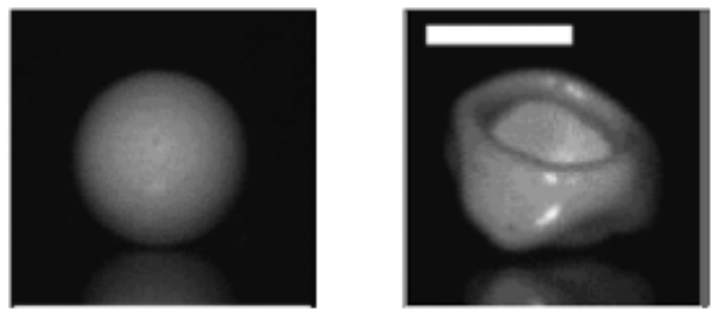

Fig. 4: Examples of grains obtained after the drying of a droplet of a nanoparticle suspension using the Leidenfrost effect. ${ }^{27}$ The scale bar represents $1 \mathrm{~mm}$.

\section{Shell formation and Péclet number}

A general agreement is found in the most recent literature regarding the mechanism leading to the buckling of drying grains. It is now well established that drying results in an accumulation of the nanoparticles at the interface of the droplet with air, which can further form a solid shell of thickness $h$, as schematised in fig. 5 .

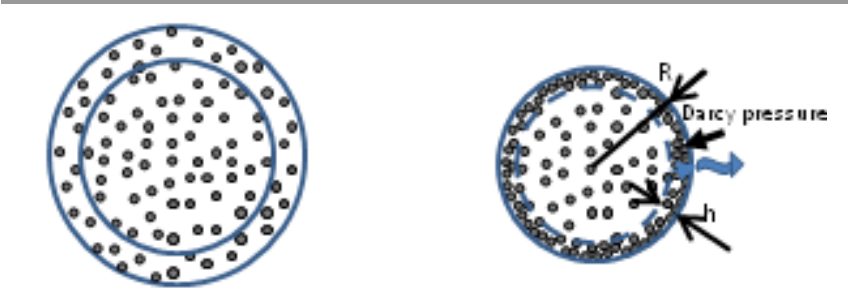

Fig. 5: Schematised droplet of a nanoparticle suspension. In the first instants of drying, the nanoparticles are homogeneously dispersed within the droplet. As shell of thickness $h$ may further form under the action of the Darcy pressure resulting from the solvent flow across the shell.

According to the conditions, the formed shell may buckle, leading to non spherical morphologies. In the literature, different experimental parameters were tuned and shown to control the onset of buckling: parameters controlling the drying kinetics, but also parameters related to the colloidal interactions between nanoparticles. For instance, increasing the drying temperature favours the apparition of buckling. ${ }^{7,10}$ Modification in the suspension formulation may also have great consequences: addition of salt favours the formation of spherical grains. ${ }^{3,8}$ In the same line, the morphology of the dried grains was found to be controlled by the addition of a polymer, ${ }^{9,28}$ namely polyacrylic acid that adsorbs onto the nanoparticles surface and is able to modify the zeta potential of the particles.

Yet the precise role of these parameters remains to be fully elucidated. For instance, Tsapis et al. ${ }^{25}$ have shown that a decrease in salt concentration, and thus an increase of the repulsion between nanoparticles, delays the onset of the buckling instability, but does not suppress it. In sharp contrast, the addition of $\mathrm{Al}\left(\mathrm{NO}_{3}\right)_{3}$ in aqueous titanium dioxide suspensions allows the formation of homogeneous spherical grains. $^{8}$

Actually, the shell formation and its mechanical properties are keys to understand what controls the onset of buckling. First, the shell is permeable to solvent and, provided an additional and denser shell of polymers or salt is not formed, it does not hinder drying. As a consequence, the volume of the droplet keeps decreasing even after the nanoparticles have accumulated in the vicinity of the air/droplet interface, which may lead to the buckling of the solid shell. In order to evaluate the shell thickness during drying, one must compare the effect of nanoparticle diffusion, which tends to homogenise the nanoparticle concentration, to the effect of drying that transports the nanoparticles to the surface of the droplet. The corresponding dimensionless number is the Péclet number that compares the drying and diffusion characteristic times, and writes for spherical nanoparticles as: 


$$
P e=\frac{\tau_{d i f f}}{\tau_{d r y}}=\frac{R^{2}}{\tau_{d r y}} \frac{6 \pi \eta \eta}{k T}
$$

Where $k T$ is the thermal energy, $a$ the nanoparticles radius and $\eta$ the solvent viscosity.

In several works it was assumed that the onset of the buckling instability is controlled by the value of the Péclet number. Yet, in spray drying conditions, for $100 \mu \mathrm{m}$ diameter droplets with $100 \mathrm{~nm}$ diameter particles suspended in water, and for a drying time of 1 second, the Péclet number is close to $5.10^{3}$. The diffusion process is thus slower by three orders of magnitudes than the drying time and the effect of nanoparticle diffusion is therefore negligible. More precisely, the droplet size above which diffusion can be neglected is given by $\left(k T \tau_{d r y} / 6 \pi \eta a\right)^{1 / 2}$ and is close to $1 \mu \mathrm{m}$ within the conditions defined above. That value is generally much smaller than the droplet size in most spray driers. As a result, all the nanoparticles concentrate in a shell, up to the maximum packing concentration. In contrast to arguments frequently found in the literature, the shape of the final grain is therefore not determined by the Péclet number, whose value remains far larger than unity in usual conditions for spray drying of colloidal suspensions. Therefore, the morphology of the final grain is determined by the mechanical properties of the shell. To account for those properties, it is necessary to consider the stress sustained by this shell, which is the object of the next section.

\section{Shell buckling and colloidal interactions}

A complete picture of the phenomena at stake during the buckling of drying droplets of colloidal suspensions was provided by Tsapis et al. ${ }^{25}$ The deformation of the shell is driven by capillary forces as menisci form between nanoparticles when solvent evaporates. Those forces are responsible for e.g. the crackings observed in drying droplets of suspensions of soft nanoparticles. ${ }^{29}$ In the case of the hard nanoparticles used in atomisation processes, capillary forces drive the buckling of the shell formed during drying as well, but the shell must first become solid in order to be able to buckle.

More precisely, the buckling instability threshold is given by the Zoelly-Van de Neut condition ${ }^{30}$ which was obtained in the frame of elastic deformations, by solving the mechanical equations for thin spherical shells. The condition relates the critical pressure $P_{\text {buck }}$ above which the shell buckles and collapses to the thickness and mechanical properties of the shell following:

$$
P_{\text {buck }}=2 E\left(\frac{h}{R}\right)^{2} \sqrt{3\left(1-v^{2}\right)}
$$

where $h$ is the shell thickness, $E$ its Young modulus, and $v$ its Poisson ratio. The suspension is incompressible, but its pressure is determined by the capillary forces at the surface. Hence, that pressure varies from room pressure - taken as zero reference - to the maximum capillary pressure as air penetrates in between the nanoparticles:

$$
P_{c a p} \approx \frac{4 \gamma}{d}
$$

where $\gamma$ is the air-suspension interfacial tension, and $d$ the diameter of the pores in between particles of radius $a$.

Assuming a pore diameter $d \approx a / 5$ the capillary pressure reaches $10 \mathrm{MPa}$ for $100 \mathrm{~nm}$ diameter particles in water. As the shell thickens, $h$ increases from zero to $R$, and the critical buckling pressure given by eq. (6) consequently increases during shell formation. The value of the modulus of ceramics suspensions - which are very rigid nanoparticles- lies between $10^{4}$ and $10^{6} \mathrm{~Pa}^{31}$. As a result, for shell thickness ranging from $h \approx 5 a$ to $h \approx R / 5$, the critical buckling pressure ranges at most from $10^{3}$ to $10^{5} \mathrm{~Pa}$. It therefore remains significantly smaller than the maximal value of the capillary pressure $P_{\text {cap }}$ during the whole drying process.

Since $P_{\text {cap }}>P_{\text {buck }}$, buckling is expected to occur as soon as the shell becomes elastic, independently of its thickness. The formation of the solid shell therefore determines the onset of buckling.

We now focus on the mechanism leading to the formation of the shell. Tsapis et al. ${ }^{25}$ have shown that the liquid to solid transition of the shell results from the viscous stress induced by the permeation of solvent through the shell. This flowinduced stress results in a uniaxal compression on the accumulated nanoparticles (fig. 5). The flow is analogous to the one through a porous medium; it results in a pressure difference across the shell that is related to flow velocity using the classical Darcy's law for porous media, and thus writes as:

$$
P_{\text {Darcy }}=\frac{\eta v}{k} h
$$

Where $\eta$ is the solvent viscosity, $v$ is the relative velocity between liquid and shell, $h$ the shell thickness and $k$ its permeability. This pressure difference is counterbalanced by the "particle pressure", or the force network that propagates from one nanoparticle to its neighbours. In the case of nanoparticles stabilized by charge-induced repulsions, the Derjaguin Landau Verwey Overbeek (DLVO) potential is used to describe those repulsions together with the attraction induced by Van der Waals forces at short range. As shown in fig. 6 , the resulting potential exhibits an energy barrier that can be overcome under the action of the Darcy pressure resulting from solvent's flow. In that case, the particles aggregate under the action of the Van der Waals attractive forces. As a result, the nanoparticles that are accumulated at the droplet's surface form a solid shell. The shell eventually buckles, as the droplet volume decreases by solvent evaporation. As pointed out in the literature, ${ }^{8,11}$ the shell is as well submitted to a Laplace pressure until the last stage of drying where the air/solvent interface penetrates into the grain. However it is the Darcy pressure that induces the aggregation of the nanoparticle and makes the shell solid-like, which is necessary to induce buckling. ${ }^{25}$

Since the DLVO energy barrier has to be overcome for buckling to proceed, a logical solution to suppress buckling would consist in increasing the amplitude of the DLVO barrier, which can be easily achieved by e.g. decreasing the ionic strength of 
the colloidal suspension. Actually, Tsapis et al. did observe that a decrease in ionic strength delays the onset of the buckling instability during the drying stage, but they could not suppress buckling.

To understand that result, a quantitative analysis has to be conducted. The permeability of the shell can be related to the the nanoparticles radius by the the semi-empirical KarmanKozeny relation that provides an expression of the permeability of a packing of spheres of volume fraction $\Phi_{c}$, following: ${ }^{25}$

$$
k \cong \mathrm{a}^{2}\left(1-\Phi_{c}\right)^{3} /\left(45 \Phi_{c}^{2}\right)
$$

Assuming that the relative velocity between the porous shell and solvent is $v \approx R / \tau_{d r y}$ yields the following expression for the Darcy pressure:

$$
P_{\text {Darcy }} \approx \frac{45 \Phi_{c}^{2}}{\left(1-\Phi_{c}\right)^{3}} \frac{\eta R h}{a^{2} \tau_{d r y}}
$$

Remarkably, the Darcy pressure increases linearly with the shell thickness, $h$. More precisely, inside the droplet, the pressure increases with the radial distance from the centre, and is maximum at the interface with air. As the droplet dries, the thickness $h$ roughly varies from a nanoparticle size $a$ to the droplet size $R$. For $100 \mathrm{~nm}$ diameter nanoparticles packed at the random close packing volume fraction, $100 \mu \mathrm{m}$ droplets and a drying time of $1 \mathrm{~s}$, the maximal Darcy pressure ranges from 100 to $10^{5} \mathrm{~Pa}$.

In summary, the flow of solvent through the shell formed by the accumulated nanoparticles results in a Darcy pressure that makes the nanoparticles come closer and may induce their aggregation. At small distances between nanoparticles, colloidal interactions, which may oppose to aggregation, must be considered. In the following we discuss the possible aggregation of nanoparticles under the action of the Darcy pressure with respect to the colloidal interaction potential in the frame of a DLVO model.

The DLVO interaction energy, that accounts for a charge induced repulsion and a Van der Waals attraction writes, in the frame of the Gouy-Chapmann model and at distances between particles surfaces $r \ll a$ as: ${ }^{32}$

$$
V_{D L V O}(r)=64 \pi k T \Gamma^{2} \rho_{i} \lambda_{D}^{2} a e^{-r / \lambda_{D}}-\frac{A a}{12 r}
$$

where $\Gamma=\tanh (e \zeta / 4 k T), e$ is the electron charge and $\zeta$ the surface potential, $\lambda_{D}$ is the Debye length, $\rho_{i}$ the ion density in the solvent and $A$ the Hamaker constant. For charge-stabilized nanoparticles, the potential reaches a maximum as $r$ increases from zero, and further goes to zero for large separation distances.

In order to account for the constant force resulting from the Darcy pressure, an effective potential can be written that includes a Darcy pressure term, such that:

$V_{t o t}(r)=64 \pi k T \gamma^{2} \rho_{i} \lambda_{D}^{2} a e^{-r / \lambda_{D}}-\frac{A a}{12 r}+\pi a^{2} P_{D a r c y} r$

As shown in fig. 6, the Darcy term results in a decrease of the energy barrier to overcome for particle aggregation to occur by increasing the minimum value of the potential much more than it increases its maximum value. The barrier threshold $\Delta V_{\text {tot }}$ for aggregation being of a few $k T$, a solid shell can form provided the Darcy pressure decreases $\Delta V_{\text {tot }}$ down to that value. The larger the DLVO energy barrier, the larger the Darcy pressure must be to reach those conditions.

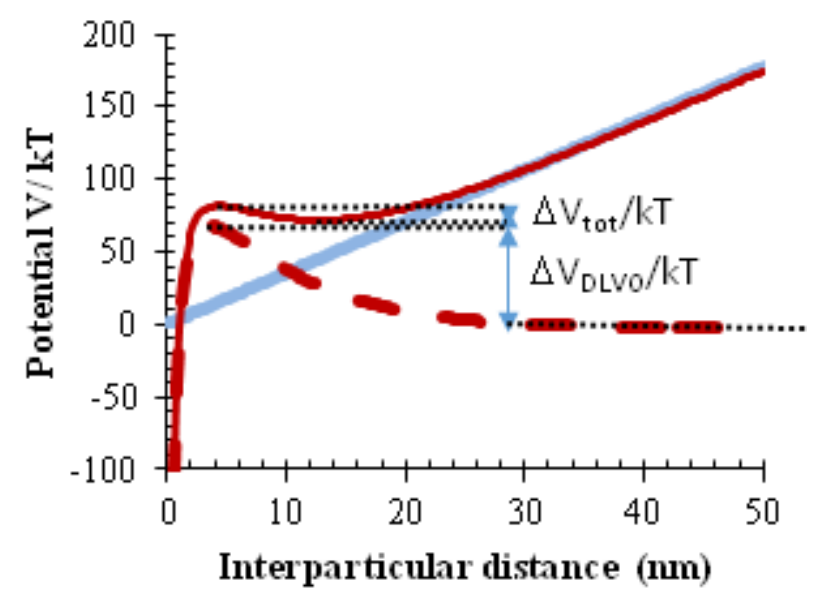

Fig. 6: Variations with the interparticle distance of the DLVO potential (dotted line), Darcy pressure potential (blue/lighter full line), and total potential given by eq. (12) (red/darker full line) for a suspension of charged colloidal particles. The effect of the Darcy pressure is to lower the energy barrier to overcome for particles to aggregate. ${ }^{27}$

The needed value of the Darcy pressure for aggregation nevertheless remains smaller than its maximum value, calculated for the more drastic conditions provided by strongly charged particles suspended in a low ionic strength electrolyte. Thus, for usual mineral suspensions for which the Hamaker constant is $A \approx 10^{-20} \mathrm{~J}$, a lower value of salt concentration $10^{-4} \mathrm{~mol} . \mathrm{L}^{-1}$, and an upper value of surface potential $\zeta=100 \mathrm{mV}$, a huge DLVO energy barrier - close to $600 \mathrm{kT}$ - is obtained, but the Darcy pressure needed to decrease that barrier down to $10 \mathrm{kT}$ is close to $6 \mathrm{kPa}$, which is more than one order of magnitude than the estimate for its maximal value. As a consequence, solid shell formation during drying cannot be avoided neither by decreasing the ionic strength nor increasing the surface potential of the nanoparticles.

The buckling time can be quantitatively predicted for given colloidal interactions: mass conservation in the droplet yields the variations of the shell thickness with time, $h(t)$. The buckling time is the time at which the Darcy pressure, which follows equation (10), is such that the energy barrier is decreased down to about $10 \mathrm{kT}$, thus allowing for particle aggregation. In that frame, the buckling time writes as:

$$
\tau_{\text {buck }} \approx \frac{\tau_{d r y}^{2} a^{2}}{100 \eta R^{2}} P_{D L V O} \frac{\Phi_{0}}{\Phi_{c}-\Phi_{0}}
$$

Where $P_{D L V O}$ is the pressure to exert to decrease the DLVO energy barrier down to $10 k T$ and $\Phi_{c}$ and $\Phi_{0}$ are respectively, the volume fraction of close packing and the initial volume fraction of the nanoparticles in the suspension.

In the case of aqueous suspensions of zirconia nanoparticles, an excellent agreement was found between the experimental values of the buckling time ${ }^{27}$ and their values predicted in that 
frame. As expected and previously observed, ${ }^{25}$ the buckling time is a decreasing function of ionic strength, as is the amplitude of the DLVO barrier. In the same line, experiments conducted with nanoparticles suspended in liquids of different viscosities showed that, in agreement with eq. (13), a viscosity increase results in decreasing buckling times. ${ }^{26}$ Remarkably, the buckling time not only depends on the colloidal interactions through $P_{D L V O}$, but also on the drying time, and thus on the drying conditions such as temperature, air flow, etc..., in agreement with reports from the literature.,

In conclusion, the Darcy pressure resulting from solvent's flow during drying is large enough to overcome DLVO energy barriers even for strong nanoparticle repulsions, and induces the formation of a solid shell made of nanoparticles. Once formed, the shell buckles since the capillary pressure is always larger than the critical pressure for buckling. Buckling is therefore expected for all initially stabilized and well dispersed colloidal suspensions. The final grain morphology will depend on different parameters such as shell thickness and mechanical properties. In addition, the volume variation induced by drying is a relevant parameter: above a threshold value of that parameter a cavity forms within the droplet, as shown in the literature. $^{33}$ In spray drying conditions, the hollow grains always buckle, preventing the formation of hollow spheres. Other routes have therefore been developed in order to prepare inorganic hollow spheres. ${ }^{34}$

According to the values of the different parameters at the onset of buckling, a large variety of shapes results from shell buckling, ranging from wrinkles to doughnut-like or deflated balloon-like, or even hollow hemispheres. ${ }^{35}$ The same shapes are observed after the drying of sessile droplets, since the same mechanisms are at stake in the latter case, provided the Pe number is large. ${ }^{36,37}$

We will see in the following section that, in contrast, buckling can be suppressed by strongly decreasing the DLVO barrier, i.e. by destabilizing the suspension. In the latter case, the obtained dried grains are full spheres.

\section{Suppression of buckling in aggregated suspensions}

We have shown above that buckling is expected for most stabilized colloidal suspensions, and that full spheres cannot be formed by increasing the DLVO barrier. Actually, Lintingre et $a .^{27}$ have shown that the relevant lever to avoid buckling is not to increase the DLVO energy barrier, but to increase the permeability of the shell to solvent. That increase is achieved by forming a suspension of aggregates of nanoparticles. The aggregates must be small enough not to increase significantly the viscosity of the suspension, but large enough to form objects much larger - and much less dense - than the individual nanoparticles. An estimate for the permeability of a packing of aggregates is provided assuming the aggregates are spherical of radius $R_{a}$, and using the Karman-Kozeny relation given by eq. (9). Thus, for a radius of ten nanoparticules radii, the Darcy pressure drops by a factor 100 and thus can remain below the
DLVO pressure. In that situation, the shell cannot form, or cannot sustain the stress if it forms, and as a result, the droplets shrink isotropically.

In practice, a decrease of the DLVO barrier is obtained by either increasing the ionic strength or decreasing the charges borne by the nanoparticle surfaces. If the amplitude of the barrier becomes of the order of $k T$, aggregates can form as the particles come closer under the action of thermal motion. The aggregation is irreversible, meaning that nanoparticles cannot spontaneously separate once they have aggregated, and the aggregates have a fractal structure, as is well known in Diffusion Limited Cluster Aggregation (DLCA) conditions. ${ }^{38}$ If the aggregation is slow enough, which is obtained with a non zero DLVO energy barrier, the size of the aggregates grows slowly with time, and suspensions of small aggregates in a reproducible state can be prepared.

Although no quantitative explanation was given, the use of slightly aggregated suspensions has been suggested by several groups in the past years in order to obtain filled spherical grains: for instance, it was found to avoid hollow shell formation in alumina particle suspensions ${ }^{3}$ and doughnut shape in titan dioxide colloidal suspensions. ${ }^{8}$ A full picture of the effects at stake combined to a quantitative study was recently provided by Lintingre et al. ${ }^{27}$ On the experimental side, they showed that the buckling time of droplets of aqueous suspensions of zirconia particles decreases as the ionic strength increases, in agreement with the description of the previous section; in addition, they demonstrated that, upon a further increase of ionic strength, the buckling time reaches a minimum value and further increases (fig. 7).

This behaviour results from the aggregation of the nanoparticles and the consequent increase of the permeability of the shell. Actually, the shell that forms from the accumulation of the fractal aggregates is very fragile and cannot sustain the stress. It consequently remains liquid-like and will never buckle. A full quantitative picture was provided by taking into account the radius of the aggregates, obtained from independent measurements on the same suspensions, in the computation of the permeability. ${ }^{27} \mathrm{~A}$ very good agreement with experimental data was obtained. Above a threshold value for the ionic strength, corresponding to threshold value of the size of the aggregates, the buckling time becomes larger than the drying time, and full spheres are formed at the end of drying as imaged in fig. 7. We emphasize that the only way to suppress buckling is to strongly decrease the shell permeability; in practice, this is achieved by slightly destabilising the colloidal suspension in order to obtain fractal aggregates, which will form highly porous shells during droplet drying. 


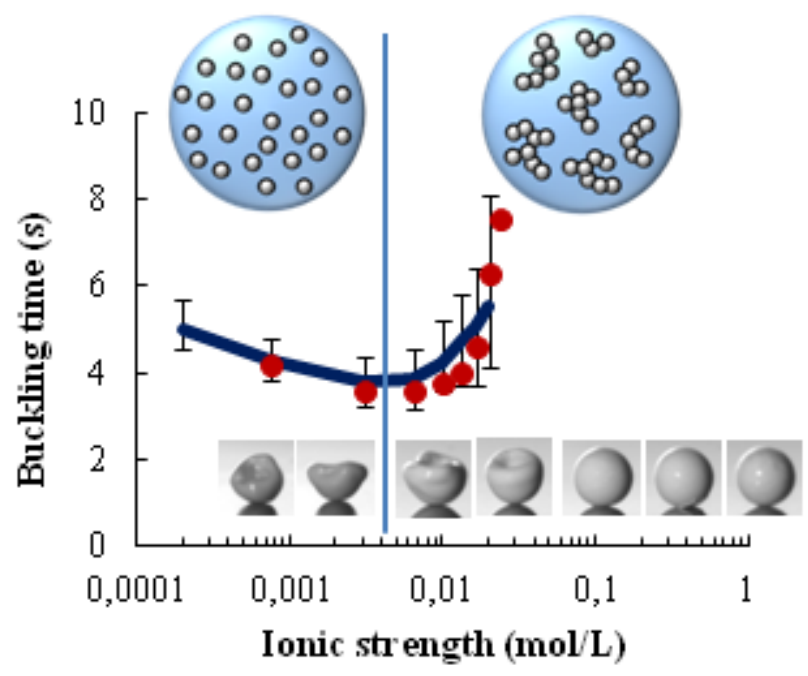

Fig. 7: Buckling time as a function of ionic strength. The experimental data measured for drying droplets of zirconia suspensions (circles) are well described by the computed times following eq. (13) (full line). The photographs show the grains obtained after full drying for different initial ionic strengths of the suspension, corresponding to different initial states (fully dispersed or suspension of fractal aggregates). ${ }^{27}$

\section{Conclusions}

In this paper, we review the different effects that have been suggested to drive the morphology of the grain/microparticles formed by spray drying nanoparticle suspensions. We show that the grain/microparticle shape does not result from hydrodynamical effects. In addition, we show the Péclet number is not the relevant critical number to consider for the spray drying of suspensions of nanoparticles. That particular point explains the successful reproduction of atomization features with single drop experiments such as Leidenfrost experiments: provided the $P e$ remains large compared to unity in the latter experiments, it is expected that similar behaviours are observed independently of the other parameters.

As schematised in fig. 8, the key parameter to prevent buckling is the permeability of the nanoparticles accumulated at the interface with air. If the suspension is initially well dispersed the permeability is small and the resulting Darcy stress large enough to overcome the DLVO energy barrier. If the suspension contains fractal aggregates, the increase in permeability prevents the shell formation and its further buckling for an aggregate size beyond a threshold values. The final morphology is therefore fully determined by the interactions between nanoparticles. The occurrence of buckling depends on the mechanical properties of the shell that results from the accumulation of nanoparticles under the action of the solvent flow.

The main conclusions summarized in this review were obtained for model colloids such as polystyrene nanoparticles or mineral nanoparticles. However these findings could be generalised to other systems: indeed food powders such as those obtain from milk exhibit strikingly similar behaviours as mineral nanoparticles. ${ }^{39-41}$ Similar morphologies are also found in pharmaceutical powders. ${ }^{42}$ In both cases similarities are not surprising. Proteins and polymers found in these products behave as colloids. Model experiments capture the physics of drying colloidal suspensions; slight differences may arise from mechanical properties of colloids themselves which are rather hard in the case of mineral nanoparticles while being softer in the case of proteins or polymers. The exact influence of colloid mechanical properties on the resulting grain/microparticle morphology should be the object of future works.

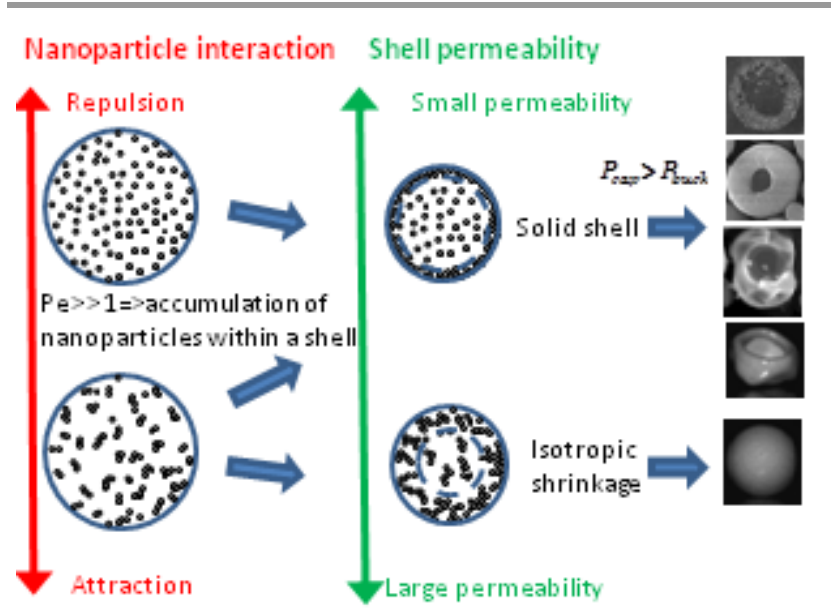

Fig. 8: Summary of the behaviours according to the nanoparticle interactions, which determine the shell permeability and consequently the Darcy pressure. The photographs are the same as in fig. 1 and 4.

\section{Acknowledgements}

Institut Galien Paris-Sud is a member of the Laboratory of Excellence LERMIT supported by a grant from ANR (ANR-10LABX-33).

\section{Notes and references}

¥ The saturation concentration depends on the osmotic pressure $\pi$ of the colloids following: $c_{\text {sat }}=c_{\text {sat }}^{0} \exp (-\pi v / k T)$ where $v$ is a molecular volume and $k T$ the thermal energy. Since the pressure $k T / v$ is of the order of $10^{8} \mathrm{~Pa}$, the exponential term is generally negligible.

1 L. Aragao-Santiago, H. Hillaireau, N. Grabowski, S. Mura, T. L. Nascimento, S. Dufort, J.-L. Coll, N. Tsapis and E. Fattal, Nanotoxicology, 2016, 10, 292-302.

2 A. B. D. Nandiyanto and K. Okuyama, Advanced Powder Technology, 2011, 22, 1-19.

3 W. J. Walker, J. S. Reed and S. K. Verma, Journal of the American Ceramic Society, 1999, 82, 1711-1719.

4 Cottrino, Y. Jorand, J. Adrien and C. Olagnon, Powder Technology, 2013, 237, 586-593.

5 M. R. Lauro, C. Carbone, F. Sansone, B. Ruozi, R. Chillemi, S. Sciuto, R. P. Aquino and G. Puglisi, Drug Dev Ind Pharm., 2016, 42, 1127-1136.

6 L. Chen, T. Okuda, X. Y. Lu and H. K. Chan, Adv Drug Deliv Rev., 2016, 100, 102-115. 
7 F. Iskandar, L. Gradon and K. Okuyama, Journal of Colloid and Interface Science, 2003, 265, 296-303.

8 S. Lyonnard, J. R. Bartlett, E. Sizgek, K. S. Finnie, T. Zemb and J. L. Woolfrey, Langmuir, 2002, 18, 10386-10397.

9 G. Bertrand, P. Roy, C. Filiatre and C. Coddet, Chemical Engineering Science, 2005, 60, 95-102.

10 R. Vehring, W. R. Foss and D. Lechuga-Ballesteros, Journal of Aerosol Science, 2007, 38, 728-746.

11 J. Bahadur, D. Sen, S. Mazumder, G. Santoro, S. Yu, S. V. Roth and Y. B. Melnichenko, Langmuir, 2015, 31, 4612-4618.

12 P. S. Grant, Progress in Materials Science, 1995, 39, 497-545.

13 P. H. Marmottant and E. Villermaux, Journal of Fluid Mechanics, 2004, 498, 73-111.

14 E. Reyssat, F. Chevy, A. L. Biance, L. Petitjean and D. Quere, Epl, 2007, 80, 34005.

15 L. A. Porritt, J. K. Russell and S. L. Quane, Earth and Planetary Science Letters, 2012, 333, 171-180.

16 S. Yamamoto and Y. Sano, Chemical Engineering Science, 1992, 47, 177-183.

17 J. A. Perdana, M. B. Fox, M. A. I. Schutyser and R. M. Boom, Chemical Engineering \& Technology, 2011, 34, 1151-1158.

18 R. Toei, M. Okazaki and T. Furuta, Drying mechanism of a non-supported droplet. First international symposium on drying, A.S. Mujumdar ed. 53-58 (1978).

19 A. L. Yarin, G. Brenn, J. Keller, M. Pfaffenlehner, E. Ryssel and C. Tropea, Physics of Fluids, 1997, 9, 3300-3314.

20 S. Basu, E. Tjerino and R. Kumar, Appl. Phys. Letters, 2013 102, 14162.

21 A. S. Gandhi, A. Saravanan and V. Jayaram, Materials Science and Engineering a-Structural Materials Properties Microstructure and Processing, 1996, 221, 68-75.

22 W. K. Rhim, S. K. Chung, M. T. Hyson, E. H. Trinh and D. D. Elleman, leee Transactions on Industry Applications, 1987, 23, 975-979.

23 J.G. Leidenfrost, De Aquae Communis Nonnullis Qualitatibus Tractatus (J. Straube, Duisburg, Germany, 1756))

24 A. L. Biance, C. Clanet and D. Quere, Physics of Fluids, 2003, 15, 1632-1637.

25 N. Tsapis, E. R. Dufresne, S. S. Sinha, C. S. Riera, J. W. Hutchinson, L. Mahadevan and D. A. Weitz, Physical Review Letters, 2005, 94.

26 G. Marty and N. Tsapis, European Physical Journal E, 2008, 27, 213-219.

27 E. Lintingre, G. Ducouret, F. Lequeux, L. Olanier, T. Perie and L. Talini, Soft Matter, 2015, 11, 3660-3665.

28 S. Tanaka, C. Chia-Pin, Z. Kato and K. Uematsu, Journal of the European Ceramic Society, 2007, 27, 873-877.

29 H. M. van der Kooij, G. T. van de Kerkhof and J. Sprakel, Soft Matter, 2016, 12, 2858-2867.

30 S. P. Timoshenko and J. M Gere, Theory of Elastic Stability (McGraw Hill, New York, 1961).

31 J. A. Yanez, T. Shikata, D.S. Pearson and F.F Lange, Journal American Ceram. Soc, 1996, 79, 2917-2924.

$32 \mathrm{~J} . \quad \mathrm{N}$. Israelachvili, Intermolecular and Surface Forces (Academic Press, New York, 1992).

33 F. Meng, M. Doi and Z. Ouyang, Phys. Rev. Lett., 2014, 113 098301.

34 J. Hu, M. Chen, X. Fang and L. Wu, Chem. Soc. Rev., 2011, 40 5472-5491.

35 C. Quilliet, C. Zoldesi, C. Riera, A. van Blaaderen, and A. Imhof, Eur. Phys. J. E 2008, 27, 13-20.

36 L. Pauchard, L. and Y. Couder, Europhysics Letters, 2004, 66, 667-673.

37 F. Boulogne, F. Giorgiutti-Dauphine and L. Pauchard, Soft Matter, 2013, 9, 750-757.

38 Kinetics of Aggregation and Gelation edited by F. Family and D. Landau (North Holland, Amsterdam 1984).
39 S. Rogers, W. D. Wu, S. X. Q. Lin and X. D. Chen, Biochemical Engineering Journal, 2012, 62, 92-100.

40 C. Sadek, P. Schuck, Y. Fallourd, N. Pradeau, R. Jeantet and C. Le Floch-Fouere, Food Hydrocolloids, 2016, 52, 161-166.

41 C. Sadek, P. Schuck, Y. Fallourd, N. Pradeau, C. Le FlochFouere and R. Jeantet, Dairy Science \& Technology, 2015, 95, 771-794.

42 R. Vehring, Pharmaceutical Research, 2008, 25, 999-1022. 